\title{
Eigentlichkeit in Heideggers Wege
}

\section{Prof. Safaa Abd Elsalam Gaafar (PhD)}

Professor der moderne und zeitgenössische Philosophie,

Philosophische Abteilung,

Universität Alexandria - Ägypten 
Missverständnie ist ein intellektuelles Schicksal, bei dem der Philosoph eine umfassende Änderung vornehmen und die Strömung in eine neue Richtung lenken möchte." Bei unserer Suche nach Heidegger haben wir seine Erfahrung von innen heraus gefunden, um auf dieses wegweisende Art seiner Philosophie zu reagieren.

Wir versuchen diese Anschuldigungen abzuwehren, die sich wie folgt zusammenfassen lässt:

(Erstens) " Heideggers Philosophie kehrt zum Alten zurück und leugnet reaktionär die Entwicklung, die in der Geschichte der Philosophie stattgefunden hat":

Es kann beantwortet werden, dass Heidegger zum Alten zurückkehrt, um aus der Erfahrung, die diese frühen Denker bewegte, zu der Quelle des Erstaunens zurückzukehren, die sie zum ersten Mal $\mathrm{zu}$ dieser Frage veranlasste. Für Heidegger ist diese Rückkehr gleichbedeutend mit der Wiederbelebung der ursprünglichen Frage nach dem Sinn des Seins, die mit ihrem Licht über Wesen überfließt.

(Zweitens) "Heideggers Philosophie hat einen negativen statischen Charakter ohne Dialektik:"

Diese Anschuldigung kann vorgebracht werden, nachdem uns gezeigt wurde, dass das Sein in- der-Welt eine dynamische Existenz ist, die dem Augenblick ein positives Sachpotential zuschreibt, das es ihr ermöglicht, mit ihren Werkzeugen, Dingen und Seienden mit der Außenwelt umzugehen.

Andererseits ist für Heidegger die Möglichkeit kein negatives logisches, sondern eine positive Möglichkeite, die das Dasein zum Eigentlichen Sein treibt. Der Kontrast, den wir zwischen dem Bild der "uneigentliche Existenz" und der "eigentliche Existenz" zeigten, offenbart eine dialektische und ununterbrochene Spannung, solange das Dasein in-der-Welt existiert.

Schließlich ist Zeitlichkeit für Heidegger auch eine Bewegung und eine ständige Spannung zwischen ihrer uneigentliche und eigentliche Bedeutung, und das Endziel dieser Bewegung ist es, die Bedeutung des Seins offenbaren. 
(Drittens) dass es in seiner Philosophie eine Tendenz zum Nihilismus und einen Aufruf zur Unterstützung des Imperialismus und des Kapitalismus gibt, und dass einige Erklärer die Verantwortung für jeden zukünftigen Krieg tragen, der Tod und Nichts hervorruft, das er immer bereichert hat.

Tatsächlich macht Heideggers Denken über die "Notlage des Zeitalters" es nicht zu einem Vorboten des Verderbens, und sein ernsthafter und tiefer Sinn impliziert keine Schwärze des Pessimismus, und das in seiner Philosophie von Nichts und Tod ein Fenster der Hoffnung, von dem aus Optimismus die Welt übersieht. Über demWesen des Menschen, da sein Potenzial höher ist als die Realität, und er immer die Form des Projekts annimmt.

Die Idee des Nichts ist für Heidegger der einzige Weg, auf dem uns das Sein offenbart wird, und diese Idee drückt nur eine der existenziellen Strukturen des ein-in -der Welt aus, und es gibt andere Strukturen, die nicht weniger wichtig sind, wie Sorge und Entschlossenheit ... usw.

Und Heidegger endet nicht mit Nihilismus; Nichts in Heidegger ist das Sein, und so öffnet zu seiner letzten Philosophie führt, die eine halbmystische und poetische Meditation über Sein, Denken und Sprache ist.

(Viertens) Dass es in seiner Philosophie eine subjeketive Tendenz und eine unmenschliche Tendenz gibt:

Tatsächlich ist Heideggers Philosophie das genaue Gegenteil aller Subjektivität, wenn für Heidegger dasSein dem Wesen des Dasein ist. Denn dies bedeutet nicht, dass dasDasein rein subjektiv ist, sondern dass es sich um eine „Ekstase" oder eine Abkehr vom Selbst handelt. Das Dasein existiert nur außerhalb von sich selbst und im Herzen des Seins im allgemeinen Sinne und in Bezug auf seine Grundstruktur ist es Sein- in-der Welt mit anderen Dasein.

Diese Anschuldigung scheint die Quelle einiger Forscher zu sein, die Heideggers Sprichwort verwirren: "Die Existenz des Daseins ist das, was es ist", und Sartres Sprichwort "Den Seinsgeht dem Wesen voraus". Das erste bedeutet, dass das Dasein in seinem Wesen keine 
reine Subjektivität ist, sondern eine Abkehr vom Selbst, und das zweite bezieht sich auf den Prozess der menschlichen Schöpfung von sich selbst Für seine Freiheit

Die "unmenschliche Tendenz" in seiner Philosophie widerspricht der Realität dieser wahren Humanismusphilosophie, solange die Menschlichkeit des Menschen auf seiner Existen znähe und seinem Gefühl der Sorge um die Formen diesers Den Seins beruht und das Hauptproblem, das ihn zu jeder Zeit wach gehalten hat, die Suche nach dem Sinn des Seins ist Mit einem Interesse an der Sprache als Werkzeug zum Ausdruck des Seinund dem klarsten Aspekt davon.

(Fünftens) "Heidegger ist der Prophet des Sentimentalismus und der Feind der Logik und Wissenschaft, und dass in seiner Philosophie eine Schwäche in der logischen Konstruktion liegt:"

Tatsächlich war die Beschäftigung mit Heideggers Denken immer das Problem der Wahrheit geblieben, und er achtete besonders auf "Logos", und er war immer damit beschäftigt, in die Tiefen des Denkens einzutauchen, und die Tiefen der menschlichen Sprache zu erforschen.

Andererseits waren die meisten seiner Werke durch strenge Denkweise und Genauigkeit des Ausdrucks gekennzeichnet, da er viele präzise philosophische Begriffe verwendete, und er war daran interessiert, den phänomenologischen Ansatz in "Sein und Zeit" insbesondere in einer strengen und präzisen Anwendung anzuwenden, die diesen Vorwurf widerlegt.

(Sechstens) "In seiner Philosophie gibt es eine nationalsozialistische Tendenz, und sein Ruf nach eigentlicher Existenz ähnelt Hitlers Ruf, der den deutschen schicksalhaften Ruf darstellt, der während seiner Regierungszeit vorherrschte, und dass er an der nationalsozialistischen Partei der Nazis teilnahm und Literatur gegen das Denken der Nazis verbrannte:"

Es ist allgemein bekannt, dass "Hitler" am 30. Januar 1933 n.chr. die Macht in Deutschland übernahm und dass lokale Zeitungen am 3. und 4. Mai 1933 die Nachricht von Heideggers formellem Beitritt zur 
nationalsozialistischen NSDAP veröffentlichten und am 27. Mai 1933 n. chr. Den Eröffnungsvortrag abhielten In seiner neuen Position als Direktor der Universität Freiburg unter dem Titel "Selbstbestätigung der Deutschen Universität" präsentierte er ein vorgelagertes Programm zur Hochschulreform.

Es wird auch festgestellt, dass er einen Streit mit den Behörden hatte; Und das liegt daran, dass "Alfred Rosenberg", der ideologische Führer des Nationalsozialismus, ihn aufforderte, zwei Professoren in Freiburg wegen ihrer Feinde des Nationalsozialismus zu entlassen, nämlich A. Wolf und A. Mülendorf und Heidegger weigerten sich, diesen Befehl umzusetzen, und reichten seinen Rücktritt von der Position des Universitätsrektors ein. Sein Rücktritt wurde akzeptiert, als er nur zehn Monate in dieser Position war, aber er hielt weiterhin seine Vorlesungen, nachdem sein schrecklicher Fehler aufgedeckt worden war.

\section{Tatsächlich wird Heidegger aus folgenden Gründen von der Anklage der Nazis freigesprochen:}

(1) Heidegger sagt in der deutschen Zeitschrift "Spiegel" über seine Wahl und seinen Beitritt zur NSDAP, dass es keine Alternative zum Beitritt zu dieser Partei in einer Atmosphäre gab, die von widersprüchlichen Meinungen unter einer großen Anzahl von Parteien geprägt ist, jedoch sein Beitritt zu dieser Partei es ist gleichbedeutend mit einem Mangel an politischem Bewusstsein oder einer "politischen Naivität", dass er Anfang der dreißiger Jahre sicherlich nicht allein war und Opfer der Täuschung durch den Nationalsozialismus wurde.

(2) Es ist notwendig zu erwähnen, dass er seine Rede nicht mit dem damals bekannten Slogan "Heil Hitler" abgeschlossen hat, sondern ihn mit dem Satz aus Platons Republik aufgenommen hat: "Große Dinge sind jetzt gefährdet." Dies ist ein Hinweis auf seinen falschen Glauben an die Richtigkeit der Behauptungen des Nationalsozialismus.

(3) Es ist möglich, diesen Vorwurf als Teil der bösartigen Kampagnen der Juden der Welt und des globalen Zionismus gegen alle zu 
betrachten, die vermuten, dass er eine Nähe oder Ferne Beziehung zum Nationalsozialismus hatte.

(4) Heideggers Beziehung zum Nationalsozialismus verschlechterte sich sehr schnell (nach zehn Monaten) und er griff den Hitlerismus nach dem Krieg an und beschrieb ihn als "eine historische Explosion konstruktiver Krankheiten mit den Besitzern des menschlichen Körpers als Ganzes, und dass es nicht einfach ist, die tödlichen Giftstoffe des Nationalsozialismus loszuwerden, und es wird lange dauern".

(5) Tatsächlich wurde Heideggers Verhältnis zum Nationalsozialismus und die Rolle als Spiel in der Hitler-Bewegung vor einem Fachgericht einer förmlichen Prüfung unterzogen, und die Untersuchung ergab, dass Heidegger kein aktives Mitglied der NSDAP war, und sein Gericht wurde vor dem deutschen Gericht freigesprochen, weil es der NSDAP angehörte bei der Umsetzung seiner Politik, die die Alliierten zwang, seine Rücksichtnahme auf ihn wiederherzustellen und ihm $\mathrm{zu}$ erlauben, wieder an der Universität zu unterrichten, nachdem all diese Anschuldigungen beseitigt worden waren.

(6) Heideggers Gedanken lieferten kein Kriterium, nach dem wir entscheiden würden, wie wir auf den deutschen Schicksalsruf reagieren sollen.

(7) Nach Angaben des Spiegel-Magazins war es damals verboten, als Direktor der Universität Studentenliteratur gegen den Nationalsozialismus vor dem Universitätsgebäude zu verbrennen.

(8) Die Geheimpolizei schrieb regelmäßig Berichte über seine Vorlesungen und Unterrichtsstunden und übermittelte sie A. Bäumler, Direktor der Universität Berlin, und E. Kriek, Direktor der Universität Heidelberg, die beide einen großen Einfluss auf die NSDAP hatten. . Einige seiner „Seminare“ wurden ebenfalls verboten, darunter ein Seminar über das Buch „Der Arbeiter“ von Ernst Junger. Sein Name wurde auf der internationalen Konferenz in "Prag" nicht unter die Namen deutscher Philosophen gesetzt, und zwei Vorträge von ihm blieben ohne Titel: "Was ist 
Metaphysik?" Und "Was ist das Wesen der Wahrheit?", und sie wurden im Geheimen verteilt.

Heidegger seinerseits entschied sich dafür, nicht mit den Behörden in Konflikt zu geraten, und war mit den öffentliche Vorträge zufrieden. Er blieb isoliert und vertieft in seine philosophischen Überlegungen im Dorf Tottenauberg im Herzen des Schwarzwaldes.

(9) Heidegger hatte ein gutes Verhältnis zu seinen jüdischen Studenten, so dass 933 jüdische Studenten bei ihm studierten, und er erwähnte eine von ihnen, eine Studentin Namens Helene Weiss, die später nach Schottland auswanderte und 1942 unter seiner Aufsicht an der Universität Basel ihre Doktorarbeit vorbereitete, die sie Dank Heidegger anerkannte. Er hatte auch eine gute Beziehung zu Jaspers und seiner jüdischen Frau, und Jaspers gab ihm alle seine veröffentlichten Bücher zwischen 1934/1938 n. chr. Seine freundschaftliche Beziehung zu seinem Professor Husserl (der jüdischer Nationalität war) hielt bis zu seinem Tod an ${ }^{(1)}$.

(Siebentens) "Heidegger ist einer der Vertreter des atheistischen Flügels der Existenzphilosophie:"

Es scheint, dass die Aufteilung der Existenzphilosophen in einen Flügel eines Gläubigen und einen atheistischen Flügel nicht viel zum tiefen Verständnis der Ansichten dieses Philosophen beiträgt, da sie eine "übermäßige Vereinfachung" offenbart, da sie eine wichtige Tatsache vernachlässigt, nämlich dass die Beziehung des Philosophen zur Religion eine Beziehung ist, die "Paradox" in vollem Umfang impliziert.

Wir können Heidegger aus folgenden Gründen nicht als Vertreter der atheistischen Seite in der Existenzphilosophie betrachten:

(1) Wenn wir uns Heideggers Existenzphilosophie genau ansehen, werden wir den Einfluss religiöser Wahrnehmungen darauf bemerken: Seine Beschreibung des Menschen erinnert uns an das Wesen, das sich mit seiner Existenz befasst, seine Konzption über die Sorge und sein Bestreben, mit dem, was die Religionen suchen, zum eigentlische Sein zu gelangen. 
Es ist bekannt, dass Heidegger aus einer katholischen Familie stammte, mit den Lehren der katholischen christlichen Religion aufwuchs, und in seiner Jugend an der Konstanzer Schule studierte, einer katholischen Schule in einem Gebiet, in dem sich Katholizismus und Protestantismus weitgehend ausbreiteten, und der Einfluss dieser religiösen Erziehung in seiner Philosophie nicht verborgen ist.

Es ist auch bekannt, dass er nach seiner Schulzeit am Jesuiteninstitut in der Stadt Feldkirch und anschließend an der Universität Freiburg mehrere Semester Theologie studierte und dass sowohl die religiöse Erziehung als auch die Neigung zur Philosophie einen starken Einfluss auf Heideggers frühes Leben hatten und er dies nicht tat Er schließt die Tür zum Dialog zwischen ihm und den christlichen Theologen, die von vielen Aspekten seiner Philosophie profitiert haben.

(2) Wir sehen in seiner Philosophie auch den Einfluss von Neuplatonismus, Augustinus, Pascal und Kierkegaard ..., der in seiner Position über Existenzelemente mystischer und ästhetischer Tendenz offenbart, die nicht geleugnet werden können, und wir sehen die Wirkung dieser mystischen Tendenz in seiner Rede über "Zeitlichkeit" in der Phase seines Denkens entwickelt.

(3) Daß Heidegger sich weigert, seine Philosophie als Atheismus zu bezeichnen, erklärt er dass "sein Gedanke sich nach der Heiligkeit vorbereitet", "die jede konzption über Gott oder Religion vorausgehen muss."

Er sagt, dass sein Vorwurf des Atheismus im Prinzip den folgenden Satz vernachlässigt, der seit 1929 in seinem Artikel über "Was ist die Ursache? gelesen wurde, in dem es heißt:

"Die ontologische Interpretation des Daseins als Sein- in - derWelt entscheidet nicht in einem negativen oder positiven Sinne, 
dass eine Person mit Gott oder mit ihm verwandt ist, und es ist unvermeidlich, zunächst zu einer angemessenen Konzeption des Daseins zu gelangen, indem die Bedeutung des Transcendenz geklärt wird - und dies ist, was ist getan hat im Seins und Zeit ,dann können wir durch diese Konzeption die ontologische Frage nach demWesen der Beziehung zwischen dem Dasein und Gott stellen" ${ }^{(2)}$.

Dieser Text kann wie folgt interpretiert werden:

A- Um das Problem der Beziehung zum Gott zu lösen, müssen wir zuerst hören und verstehen, ausgehend von der Realität des Seins, denn Sein ist das Schicksal des Denkens, und was heilig ist, und das verstehen wir ausgehend von diesem göttlichen Wesen, anhand dessen wir den Inhalt der Idee von Gott bestimmen können.

B- Dass Heidegger kein Leugner der Gottheit ist, denn wenn sich die Philosophie des Seins zu einer Ontologie entwickelt, schlägt sie neue Möglichkeiten für die Idee Gottes vor, und diese Möglichkeiten sind möglicherweise weiter entwickelt als das traditionelle Konzept der Gottheit, und sie erinnern uns an den Gott, von dem Meister Eckhart sprach (1260 - 1327/28). Und erinnern Sie uns an den Gott, der nach Paul Tellich Konzption.

So kann argumentiert werden, dass Heideggers Schriften über die Existenz ein undeutiger Versuch sind, nach Gott zu suchen, und ein "überzeugender" Ausdruck über den Fluch des Glaubens an Den SeinsGottes, und dass seine schwierigen, mehrdeutigen Ausdrücke die gleichen alten Einstellungen zur Religion verbergen, und Beispiele dafür werden in seiner Rede über Entschlossenheit und seine religiöse Bedeutung deutlich: Er hat die Entschlossenheit, das Projekt zu erfüllen, und das Gewissen in der Religion ist der Weg, um Sünde zu vermeiden und die Schuld der Seele auszudrücken, aber für Heidegger ist es der Weg zur eigentliche Existenz, und der Tod in der Religion ist eine unvermeidliche Realität, und für Heidegger ist es die Beschleunigung der Verwirklichung des Potenzials und 
die Ewigkeit in Religion hat sich in eine Ekstase zur Zeitlichkeit des Daseins in- der-Welt verwandelt.

C- Wenn Heideggers Denken in "Sein und Zeit" weit von der Theologie entfernt zu sein scheint, bedeutet dies nicht seine Bekehrung zum Atheismus, und dies erinnert uns an Kants Position in der "Kritik der Reinen Vernunft", als er "vorübergehend" die Erforschung von Religion und Metaphysik ausschloss, ohne Atheist zu sein.(3)

D- Mann kann sagen, dass Heideggers Denken in diesem Zusammenhang über das „Heilige“Die Gottheit und der Gott oder die Götter unterteilt ist und der Treffpunkt dieser Spaltungen „Wahrheit als Erleuchtung des Seins“ ist.

In Heideggers Denken die Beziehung zwischen Mensch und Gott nicht auf der Prämisse, dass der Mensch ein "denkendes Tier" ist. Es ist vielmehr eine im Wesentlichen Existenz, die zum Sein im Sinne eines "Ereignis " gehört.

Er stellt fest, dass Heideggers Frage nach ,dem Heiligen. und der Gottheit. und Gott" im Rahmen der Erleuchtung des Seins Ausdruck seiner Vorstellung von der „Nähe und Abwesenheit Gottes" ist: Der Mensch ist von dem Seinweggegangen und hat sich darauf konzentriert, zu vergessen, was er ist, und wenn er sich der Dimension des „Heiligen“ nähert, braucht er die Wahrheit des Seins, weil sie nur dadurch sichtbar wird, was bedeutet, dass das Denken über die Erleuchtung des Seins den Weg für den möglichen Zugang zum Heiligen durch "das Licht des Seins" ebnet.

Auf dem anderen Seite beschäftigte sich Heidegger mit den Konzepten der Hölderlin, der Heimatlösigkeit und der Heimat, durch die „Werke der Hölderlin-Dichtungen“, die die Fragen vermieden, die Heidegger in der Ontologie hängen ließ. Die Heimatlösigkeit drückt die Position des modernen Menschen in seiner Distanz zum Seinsaus, und das Wohnen ist Ausdruck seines Ansehens Nähe dem Sein, denn in der Heimatlösigkeit beginnt das Vergessen der Seiende des Seins und das Verbergen der Wahrheit, so dass der Seiende zu einer bloßen 
Sache inmitten der Dinge wird und zu einer Welt gehört, in der es nach der Heiligkeit abwesend ist, und die Überwindung dieser Heimatlösigkeit beginnt zu existieren, wenn sie wieder erleuchtet wird, und wir wissen es im Licht der Wahrheit.

Daraus wird deutlich, dass „das Heilige“ die Bedeutung ist, in der es nach Göttlichkeit und Gott inkarniert wird, und dass der „ontologische Differenz" zwischen Sein und Seinende analog zu einem anderen „theologischen Differenz" ist, der besagt, dass Gott nicht existiert und es nicht Den Seinselbst ist, und dass es sich von dem Sein im Sinne des Ereignis unterscheidet und dass es in das Vorhältnis der Wharhei im Bild des Ereignis gezeigt ${ }^{(4)}$.

(4) Heideggers Philosophie ist eine historische Suche nach dem Sein, die hauptsächlich eine religiöse Forschung ist. Heidegger sagt: "Wir müssen von dem Sein an Gott denken".

\section{Die Interpretation dieses Satzes lautet wie folgt:}

- "Das Sein ist nicht Gott", weil Sein ist die Bedeutung der Geschichte, , und dieses Sein kein Subjekt, kein Objekt, keine Person oder ein Ding ist, sondern eine reine Offenheit und eine reine Beziehung, und außerhalb seiner Beziehung zum Menschen gibt es nichts.

- Das Sein wird nicht in Bezug auf Effektivität gedacht, sondern in Bezug auf Präsenz und Immanenz.

- Dass die Aufgabe des Denkens und der Theologie darin besteht, sich vom Bild des Gottes der Philosophen zu befreien, um sich dem wahren Gott zu nähern, indem man das Sein in Frage stellt.

- Dass Gott nicht der Wert ist ", das ist die Wahrnehmung, die in der westlichen Philosophie von Platon bis Nietzsche vorherrschte.

Und - dass Gott nicht gestorben ist und dass seine Gottheit lebt. Es ist näher am "Denken" als am Glauben und erhält seinen Ursprung aus der Realität dem Sein.

- Dass Gott keine Person ist, sonst werden wir auf die Idee von Gott ein existenzielles Sprichwort anwenden, das nur in der 
Anthropologie vorkommt.

Das Ergebnis ist, dass Gott - nach Heidegger - weder eine Seiende noch einePerson ist, und seine Existenz die Ablehnung jeder Rechtfertigung und jedes Beweismaterial zum Ausdruck bringt.

(5) Heidegger hat in diesem Zusammenhang ein sehr wichtiger Satz, in dem er sagt: "Nur noch ein Gott kann uns retten. "

\section{Dieser Satz kann wie folgt interpretiert werden:}

A- Dass die Fundamentale Ontologie von "Sein und Zeit" eine "Theologie" offenbart, die zum Beispiel in diesem "Fall" deutlich wird, beschreibt die Bewegung in Richtung der Welt der Dinge und Werkzeuge und nimmt daran teil, so dass das im vorherigen Satz enthaltene verb "retten" oder "speichern" ein Ausdruck der Bewegung in Richtung ist im Gegensatz dazu drückt das eigentlische Sein"die Möglichkeit des Sein" aus, und das Wort "Möglichkeit" drückt die Differenz zwischen Sein und Seiende positiv aus, und "Denken" unterstützt diese Differenz zwischen dem Möglichen und der Realität und repräsentiert die Spannung gegenüber dem Seinden.

B- dass es ein verhältnis zwischen dem theologischen Differenz zwischen Gott und Seiende gibt - ohne den göttlichen Differenz hätten die Seienden keine Bedeutung - und Heideggers ontologischen Differenz zwischen Sein und Seinenden, und diese Differenz begründet jede Differenz im Seienden, so wie diese Differenz im Fall des Eigentliche Sein nur durch die Präsenz Gottes erreicht wird.

Wir schließen daraus, dass der Sinn des Dseins für Heidegger mit "Gott" zusammenhängt und dass seine Ontologie nicht von Anfang an auf Theologie basiert, um nicht unter die traditionelle Metaphysik zu fallen, die sich seiner Meinung nach zwischen Sein und Seiende vermischt.

Die Theologie ist in Heideggers Ontologie immanent, und der Sinn des Seins folgt der Idee Gottes, und dadurch wird die Idee 
der „Erlösung“ oder „Retten“ formuliert Sondern Sein und Seinende wird mit der Idee Gottes geretten werden.

Es stellt fest, dass Heidegger das Wort "Gott" in einem identifizierbaren, singulären und einem Plural Form verwendet hat, um damit seine neue Verwendung des Wortes $\mathrm{zu}$ beabsichtigen, mit dem er das alte "metaphysische Idol" überschreitet und es seiner formalen Formen beraubt, während er die Bedeutung von "der Einzigartigkeit der Heiligkeit" beibehält.

Er merkt auch an, dass die Bedeutung der Theologie in seiner Idee des "Entschlossenheit", die das Ziel des Denkens ist, und bei jedem Schritt der Erneuerung dieses Ziels auf ihn beschränkt ist.

C- Der Satz zeigt auch an, dass "Retten" für uns durch reines Denken erreicht wird, nicht durch Notwendigkeit an einen Gott denken, weil das Denken immer Gott zu verdanken ist und nicht unbedingt an Gott denkt, und dies drückt die Bedeutung von Heideggers schwere Denkweise aus.

Für ihn zu denken ist die doppelte Handlung des Seinseinerseits und des Daseins andererseits, drückt eine aufrichtige Rezeption aus: "Wir versuchen nicht zu denken, sondern das Denken kommt zu uns. ".

D- Es zeigt auch an, dass das Wort "Sein" den Ort der Verwirrung im metaphysischen Erbe zwischen Seinund Seinde anzeigt, und das ist das "Verfallen" in Heideggers Konzept und die Gegenbewegung zu diesem Verfallen, die Heidegger - wie in der Phrase genannt wird und das verb "retten" bezieht sich hier auf die Überwindung der Illusion der Existenz, die als Realität wahrgenommen wird, auf die Realität des Seins als Gedanken, und Gott erlaubt uns zu denken, und dass die eigentliche Gedankenerfahrung eine Bedingung ist, die es uns ermöglicht, Gott $\mathrm{zu}$ begegnen, und diese Theologie ist untrennbar mit unserer menschlichen Natur verbunden, die in Aktion dargestellt wird, nicht mit Theorie oder Bestreben zum 
eigentlichte Sein und darin werden wir durch Gott befreit. Und die "Frage nach dem Gott" wird nur durch die "hermeneutische Frage nach dem Sinn des Seins" verstanden,.

Heidegger - damals - theologisch im Prinzip und am Ende. Das Wesen des Christentums kann erklärt werden, nachdem er es als "Gedankenfreiheit" neu definiert hat, und Heidegger sagt in seinem Buch "Unterwegs Zur Sprache "Ohne die theologische Zuflucht, hätte ich den Weg des Denkens nicht erreicht".

Die im vorherigen Satz erwähnte „Befreiung“ ist ,erlauben zu denken" und ist weniger eine Rettung aus einem gegenwärtigen Zustand als vielmehr ein Überleben aus einem andauernden Zustand. Wir sind immer in einem Zustand zu einem Gott, um denken zu können; Denken ist die Aufgabe, die menschliche Natur zu überwinden. Es ist vielmehr ein Merkmal, das Sein auszeichnet - oder vielmehr - es ist das Verhältnis des Seins zum Dasein.

Daraus wird klar, dass Denken das Geschenk des Seins ist, und wir müssen uns für dieses Geschenk bedanken(5).

(5) Heidegger sagt in einer seiner Interpretationen von "Hölderlin" oder "dem Dichter der Zukunft", wie er es sieht: "Unsere Zeit ist eine Zeit der Not, weil sie unter dem Einfluss zweier Arten von Enteignung steht. Es gibt keine Götter mehr, wie Nietzsche sagte, und was nach dem neuen Gott kommt, und der extreme Mangel an Gott ist aber Ruf und Versprach" .

Heidegger meint damit, dass das Zeitalter, in dem es keine Götter mehr gibt, das Zeitalter ist, in dem die Bedeutung der Seinsfrage aufgehoben wurde, und dass die Suche nach ihrer Bedeutung unvermeidlich ist und diese Bedeutung nicht "vollständig" erreicht werden kann, weil sie weit bis zum Maximum ist, und dieser Weg dorthin ist der Weg, den wir finden unsere Identität und damit verbinden wir uns mit uns selbst, öffnen uns des Seins und behalten unsere Nähe dazu.

Wir können uns "mit Denken" und "Dichtung" auf die Entstehung oder den Untergang Gottes vorbereiten, und wir leben jetzt in der 
Ära der Abwesenheit von Göttern, und wir können nicht an Gott denken; Vielmehr können wir uns höchstens darauf vorbereiten, darauf zu warten, und in uns selbst die Vorbereitung auf dieses Warten wecken.

Heidegger ging in seinem fortgeschrittenen Denkweg davon aus, dass durch die Abkehr von der Metaphysik und Ontologie vorherrschenden Theologie im traditionellen Sinne seine religiöse Dimension zu erscheinen beginnt, und dass er eine spezielle Sprache verwendete, um Nietzsches Gedanken einerseits und Hölderlins Gedichte andererseits zu interpretieren.

"Was Nietzsche betrifft", glaubt er, dass die Idee einer Umwertungaller Werte nach Nietzsche nichts anderes als ein oberflächliches Verständnis von Nietzsches Absicht ist, und dass das Wichtigste "der Mensch als Gesetzgeber der Werte und deren Schöpfer" ist. Was Nietzsche als "den kommenden europäischen Nihilismus" verstand, verstand Heidegger nicht als Bedeutungsverlust von Werten, sondern als Ende der alten Werteliste, und er nannte das den Begriff "des Seinvergessens."

Was Hölderlin betrifft, hat Heidegger es wiederentdeckt. Wenn der letzte die Götter in der Antike für Hölderlin der christliche Gott war, und wir jetzt in der Ära der Abwesenheit der Götter leben, dann ist Heideggers Sein mehr als eine bloße Präsenz geworden, es ist eher eine "Abwesenheit", "weil die Natur gerne verschwindet", sagte Heraklit, und es ist kein Geheimnis, dass dieser Satz uns zum Warten einlädt, wie Hölderlin uns nannte.

Dieses Denken ist nicht länger ontologisch oder theologisch im traditionellen Sinne. Während Hölderlin, die Frage "Wer ist Gott?" eine der schwierigsten Frage, interpretiert,werist Gott aufgrund unserer unzureichenden Art, über ihn zu sprechen, nicht erreichen können und nur fragen können: " Wer ist Gott? Was die Heiligkeitsdimension und das Heilige in seinem Denken anzeigt, sagt er in diesem Zusammenhang: Der Verlust der Heiligkeitsdimension und des Heiligen ist die höchste Gefahr unserer Zeit.

Heidegger schrieb 1921 in einem persönlichen Brief an Karl 
Löwith einen seiner Schüler und Freunde:

"Vielleicht ist einer der schwerwiegendsten Fehler, dass Sie und Becker mich als Philosoph wie Nietzsche und Kierkegaard und andere gesehen haben ... und ich kann sagen, dass ich kein Philosoph bin, aber es gibt keinen Vergleich zwischen mir und ihnen, weil ich ein christlicher Theologe bin".

Was in dieser wichtigen Rede erwähnt wurde, weist auf Folgendes hin:

Die tiefe Motivation, die Heidegger auf seinem intellektuellen Weg antreibt, ist "Theologie": Er versteht sich als christliche Theologe, und dies bedeutet, dass alle Fragen, die er gestellt hat, auf die Befreiung von der Theologie im traditionellen Sinne abzielen, auf der er aufgeworfen wurde, damit er ein eigentlichter christ sein kann, wenn er eine neue Mission annehmen möchte. Von der Theologie im Gegensatz zum vorherrschenden theologischen Christentum, und ihm wurde dabei geholfen, sagte er, was er aus den Lehren an der theologischen Fakultät der Universität Freiburg gelernt hatte.

Diese neue Aufgabe ist in der „Befürwortung und dem Schutz des Glaubens" ${ }^{\text {(6) }}$ zusammengefasst, und dieser Satz wurde $1923 \mathrm{n}$. chr. von „Gadamer“ im Dialog mit Heidegger über die Theologie als Denkaufgabe gehört, und ihm wurde klar, dass die Faktizität des Daseins, das sich mit dem Sein besorgt, der richtige Anfang für die Verwirklichung dieser Auf gabe ist.

Es ist klar zu sagen: Die "Frage nach dem Sein", die Heidegger immer zu provozieren versuchte, und der Versuch, sie zu beantworten, ist am Ende und in seiner neuen Form nichts anderes als die "Frage nach dem Gott". 


\section{Referenzen:}

1) Spiegel, " Gespräch mit Heidegger am 23-9-1966, Aus Spiegel 31-5-1976, S. 196-204

2) Heidegger, M.: "The Essence of Reason", Abilingual ed. In Coporating the German Text "Von Wesen des Grundes" trans. By Terrence, T. Malick, Northwestern University press U.S.A, 1969. P. 91.

3) Hermann, Fr. W. Von. : "Prosminar über (Sein und Zeit)" Protokoll der 1 . Sitzung am 18-10-1990 Von A. Stützer, S. 7

4) Hermann Fr. W. Von. : "Das Heilige, Die Gottheit und der Gott im Seinsgeschichtlichen Denken Heideggers" Freiburg I. Br. , 1990, S.8-10

5) Crettela, Henri " La Theologie de Heidegger in Heidegger Studies" Dunker Humbolt, Berlin Vol. 5, 1990 P. 11-25

6) Gadamar , Hans Georg " Die Religiose Dimension" , 1981 in "Heideggers Wege" - Studien zum Spätwerke, J.C.B mohr (Paul Siebeck), Tübingen, 1983, S 140-151 